\title{
Design of sub-Angstrom compact free-electron laser source
}

Rodolfo Bonifacio $^{a}$, Hesham Fares ${ }^{a, c, *}$, Massimo Ferrario ${ }^{a}$, Brian WJ McNeil ${ }^{b}$, Gordon RM Robb ${ }^{b}$

a INFN-LNF, Via Enrico Fermi, 40 - 00044, Frascati (Roma), Italy

${ }^{\mathrm{b}}$ SUPA, Department of Physics, University of Strathclyde, Glasgow, G4 0NG,UK

${ }^{c}$ Department of Physics, Faculty of Science, Assiut University, Assiut 71516, Egypt

*hesham.fares@Inf.infn.it; Tel: +34 7287 1745; FAX: +39069 4035281

KEYWORDS: Free electron Laser; Quantum Free electron Laser; Compton Backscattering; x-ray

\begin{abstract}
In this paper, we propose for first time practical parameters to construct a compact sub-Angstrom Free Electron Laser (FEL) based on Compton backscattering. Our recipe is based on using picocoulomb electron bunch, enabling very low emittance and ultracold electron beam. We assume the FEL is operating in a quantum regime of Self Amplified Spontaneous Emission (SASE). The fundamental quantum feature is a significantly narrower spectrum of the emitted radiation relative to classical SASE. The quantum regime of the SASE FEL is reached when the momentum spread of the electron beam is smaller than the photon recoil momentum. Following the formulae describing SASE FEL operation, realistic designs for quantum FEL experiments are proposed. We discuss the practical constraints that influence the experimental parameters. Numerical simulations of power spectra and intensities are presented and attractive radiation characteristics such as high flux, narrow linewidth, and short pulse structure are demonstrated.
\end{abstract}




\section{Introduction}

The development of a compact, tunable, near monochromatic hard $x$-ray source has wideranging applications in modern medical, commercial, and academic research applications e.g. microscopy and nuclear resonance absorption. Some properties of the $\mathrm{x}$-ray radiation which are important for these applications are narrow spectral width, high photon flux, high-brightness, pulse duration (in the ps to fs range) and variable polarization.

A potentially attractive ultra-short $x$-ray source has been proposed by replacing the magnetic undulator in the conventional undulator Free Electron Laser (FEL) by ultra-high intensity laser pulses (a laser undulator) [1,2]. Synchrotron radiation is emitted when an electron beam of substantially lower energy interacts with a counter propagating laser undulator. The Compton backscattered process can be treated as that of an undulator FEL operated in the Self Amplified Spontaneous Emission (SASE) mode [2,3]. The quantum regime of SASE FELs is characterized by a dimensionless parameter $\bar{\rho}=\rho(\gamma m c / \hbar k)$, where $\rho$ is the classical FEL parameter, $\gamma$ is the electron energy (in rest mass units), $m$ is the electron mass, and $k$ is the photon wavenumber [4-6]. Since the maximum induced energy spread $\Delta \gamma \sim \rho \gamma$, hence, $\bar{\rho}$ is understood as the ratio between the classical momentum spread $\Delta \gamma m c$ and the photon recoil $\hbar k$. The quantum effects become apparent when $\bar{\rho}<1$ where the discreteness of momentum exchange becomes significant. In the quantum regime, the broad and spiky classical spectrum is converted to discrete lines due to a distinct transition between two energy levels. This process is called quantum purification. The width of this single emission line in the quantum FEL (QFEL) is $(\Delta \omega / \omega)_{Q F E L}=\lambda_{r} / L_{b}$ [7] where $\lambda_{r}$ is the radiation wavelength and $L_{b}$ is the electron bunch length. In the classical regime when $\bar{\rho}>1$, many momentum states are occupied and a multi-frequency spectrum with equally spaced lines is observed. The spectral width of the classical FEL (CFEL) spectrum is given by $(\Delta \omega / \omega)_{C F E L} \sim 2 \rho$ with the average number of spikes $N_{s} \sim 2 \rho /\left(\lambda_{r} / L_{b}\right)$ [8].

Recent advances in laser-plasma accelerators have demonstrated generation of low energy spread, low-emittance, and very short electron bunches (fs duration) $[9,10]$. On the other hand, present day lasers (e.g., Nd:YAG/Nd:glass lasers) can provide laser pulses with high peak power (TW range) and relatively long pulse duration (ns-ps range). These characteristics create a possibility to realise an efficient $x$-ray QFEL using a Compton backscattering configuration. Such an $x$-ray QFEL would assist in observing fundamental processes that require fs time resolution and atomic scale spatial resolution. The narrow bandwidth of longer laser undulator pulses is required to achieve a narrow $x$-ray bandwidth. It is noted that a basic requirement to realise a QFEL is a high quality electron beam with a low normalized emittance and a sufficiently small energy spread. This can be achieved using a low charge per bunch (e.g., using short electron bunches with high repetition rate). 
In this paper, we present design studies for a compact sub-Angstrom radiation source based on the Compton backscattering configuration. The criteria and constraints that are inherent in the requirements for all experimental parameters are discussed. We use the working equations of FELs in the Compton regime discussed in Ref. [7] to propose experimental parameters of $\leq 0.5 \AA-$ QFELs. In this paper, we assume operation in the quantum regime taking $\bar{\rho} \ll 1$. The quantum properties of the radiation such as full temporal coherence are demonstrated. It is confirmed that the experimental parameters of QFELs are attainable using current electron beam and laser undulator technologies.

\section{Basic formulas for QFEL experiments}

In this section, we present the basic formulae of the laser-electron Compton backscattering scheme (i.e., Quantum SASE FELs). We also discuss the design criteria for the QFEL experiments.

In the Compton backscattering process, the radiation wavelength $\lambda_{r}$ is identical to that of an undulator FEL by replacing the undulator period by half of the incident laser wavelength $\lambda_{L}$ and the undulator parameter $K$ by the dimensionless laser strength parameter $a_{0}=|e| \bar{A} / m c^{2}$, where $\bar{A}$ is the laser vector potential. The resonance radiation wavelength is then [7]:

$$
\lambda_{r}=\frac{\lambda_{L}}{4 \gamma^{2}}\left(1+a_{0}^{2}\right) .
$$

The laser undulator parameter $a_{0}$ is given in terms of incident laser power $P$ by

$$
a_{0}=\frac{2.4 k_{L} \lambda_{L}}{R_{0}} \sqrt{P(T W)} .
$$

where $R_{0}$ is the minimum radius of the laser and $k_{L}$ is 1 or $\sqrt{2}$ for a Gaussian or for a flat top transverse profile of the laser. It is noted that the position-dependent laser radius $R(z)$ varies due to the intrinsic Gaussian beam diffraction. If the laser diffraction is taken into account, the laser parameter $a_{0}$ shown in Eqs. (1) and (2) should be replaced by $a(z)=a_{0} /\left[1+\left(z / Z_{L}\right)^{2}\right]$ where $Z_{L}$ is the laser Rayleigh range. When $a_{0} \gtrsim 1$, the resonance wavelength given by Eq. (1) becomes spatially dependent broadening the emission spectrum. One important criterion for QFEL optimization is to fulfill the condition $a_{0}<1$ to overcome the laser diffraction and resultant spectral broadening. From Eq. (2), the constraint $a_{0}<1$ imposes a limit on the maximum laser undulator power, as discussed later. The diffraction effect can be reduced by guiding the laser pulse in a plasma-filled $[11,12]$ or vacuum $[13,14]$ capillary waveguide or by means of energy chirp in the electron pulse [15]. However, it is beyond the scope of this paper to treat in detail the means of negating the laser diffraction effect.

As discussed above, the transition from the classical to quantum regimes is determined by the quantum FEL parameter $\bar{\rho}$ that is related to the classical FEL parameter $\rho$ given by:

$$
\rho=\bar{\rho} \frac{\hbar k}{\gamma m c}=\frac{1}{2 \gamma}\left(\frac{I}{I_{A}}\right)^{1 / 3}\left(\frac{k_{e} \lambda_{L} a_{0}}{4 \pi \sigma}\right)^{2 / 3} .
$$


where $I$ is the peak current of electron beam, $I_{A} \approx 17 \mathrm{kA}$ is the Alfven current, $\sigma$ is the rms electron beam radius, and $k_{e}$ is 1 or $\sqrt{2}$ for a Gaussian or for a flat top transverse current profile respectively. Using Eq. (3) with Eq. (2), we can write the peak electron current as:

$$
I(A)=50 \bar{\rho}^{3} \frac{R_{0}^{2} \sigma^{2}}{k_{e}^{2} k_{L}^{2} \lambda_{L}^{4} \lambda_{r}^{3}} \frac{1}{P},
$$

where $\lambda_{r}$ is in $\AA, \lambda_{L}$ is in $\mu m, R_{0}$ is in $\mu m, \sigma$ is in $\mu m$, and $P$ is in $T W$. In Eq. (4), it is noted that $I \propto \bar{\rho}^{3}$ and $I \propto 1 / \lambda_{r}^{3}$. Therefore, the peak current required in the FEL experiments operated in the quantum regime $(\bar{\rho}<1)$ is much smaller than that required in the classical regime $(\bar{\rho}>1)$. This fact indicates the important role of the quantum regime to realize a compact $\mathrm{FEL}$ experiments in the $x$-ray range.

In the classical regime, the gain length $L_{g}$ and the cooperation length $L_{c}$ for the field growth are written as [8]:

$$
L_{g}=\frac{\lambda_{L}}{8 \pi \rho}, \quad L_{c}=\frac{\lambda_{r}}{4 \pi \rho} .
$$

It is considered that the interaction length $L_{\mathrm{int}}$ is the saturation length $L_{\text {sat }}$ and is given by the laser pulse duration $c \tau_{L} . L_{\text {int }}$ is also determined by a factor $n_{i}$ times $L_{g}$, i.e.

$$
L_{\text {int }}=L_{\text {sat }}=c \tau_{L}=n_{i} L_{g} \text {. }
$$

where $n_{i}$ is the number of $L_{g}$ in the interaction length $L_{\text {int }}$.

for the lasers with Gaussian profile

The spectral width due to the finite time of emission when the interaction length $L_{\text {int }} \gg L_{\mathrm{b}}$ is given by [7]

$$
(\Delta \omega / \omega)_{Q F E L} \approx \frac{\lambda_{r}}{L_{b}} .
$$

The normalized beam emittance must satisfy the restrictive condition [7]:

$$
\varepsilon_{n} \leq \sigma \sqrt{\Gamma\left(1+a_{0}^{2}\right)}
$$

where $\Gamma$ is the FEL line width equal to $\rho \sqrt{\bar{\rho}}$.

In the quantum regime, as the peak (or saturation) power of the FEL radiation is approximated by [7]:

$$
P_{r} \approx \frac{I}{e} \hbar \omega
$$

the number of emitted photons per pulse is:

$$
N_{\mathrm{ph}} \approx \frac{Q}{e}
$$

where $Q$ is the electron bunch charge. 


\section{Proposal design for sub-Angstrom QFEL experiments}

\subsection{Criteria and constraints}

In the classical regime (i.e., when $\bar{\rho} \gg 1)$, the number of spikes is $N_{s} \sim 2 \rho /\left(\lambda_{r} / L_{b}\right)=$ $L_{\mathrm{b}} /\left(2 \pi L_{c}\right)$. In the quantum regime when $\bar{\rho}<1$, the chaotic temporal structure of the radiated pulse is reduced. In this study, we assume $L_{\mathrm{b}} \gg L_{c}$, so that many radiation spikes would occur in the classical regime. Therefore, when $\bar{\rho}<1$, the improved spectral characteristics of the QFEL interaction should become apparent.

To determine the experimental parameters, we begin by choosing the radiation wavelength $\lambda_{r}$, the laser wavelength $\lambda_{L}$, and an appropriate value for the quantum regime of $\bar{\rho}=0.2$. For saturation, we choose $n_{i} \sim 24.4$ (see figures 3 and 4 ). It is noted that for smaller values of $\bar{\rho}$ (i.e., $\bar{\rho}<0.1$ ) impractical values for the gain length $L_{g}$ and the laser pulse width $\tau_{L}$ are necessary. Then, the energy of the laser pulse $U=P \tau_{L}$ become more challenging. For values of $\bar{\rho} \geq 0.4$, the quantum properties of the FEL interaction are greatly reduced.

Using realistic values for the laser power $P$ and the laser parameter $a_{0}$, we can determine the parameters $\gamma$ using Eq. (1), $R_{0}$ using Eq. (2), $\rho=\bar{\rho}(\hbar k / \gamma m c), L_{\mathrm{c}}$ and $L_{g}$ using Eq. (5), $\Gamma=\rho \sqrt{\bar{\rho}}$, and $\tau_{L}$ using Eq. (6). In this work, $\sigma$ is varied from 0 to $R_{0}$. The normalized emittance,

$\varepsilon_{n}=\sigma \sqrt{\Gamma\left(1+a_{0}^{2}\right)}$ is assumed in Eq. (8) and varies linearly with $\sigma$. Note that for high efficiency operation, to maintain beam/laser overlap, $\sigma<R_{0}$. By varying $\sigma$, the current $I$ is also varied according to Eq. (4) and the corresponding radiation power $P_{r}$ is obtained using Eq. (9). As will be shown later, to satisfy the relation $\varepsilon_{n}=\sigma \sqrt{\Gamma\left(1+a_{0}^{2}\right)}$, while choosing reasonable values for other parameters, $\sigma$ will be in the order of one-tenth of $R_{0}$. Therefore, in lasers with a Gaussian profile, the intensity distribution can be assumed uniform across the beam radial profile.

The values of $P$ and $a_{0}$ are then chosen to satisfy the following criteria: (i) $a_{0}<1$ to minimize the laser radiation wavelength in Eq. (1) and the emittance given by $\varepsilon_{n}=\sigma \sqrt{\Gamma\left(1+a_{0}^{2}\right)}$, (ii) $n_{i} \sim 24.4$, so that saturation can be attained for the power growth, (iii) the current $I$ should have realistic values in the working range of $\varepsilon_{n}$ (i.e. $I<1 \mathrm{kA}$ ) (Note that the applicable range of $\varepsilon_{n}$ is determined by the applied range of $0<\sigma \leq R_{0}$ ), (vi) The laser pulse duration $\tau_{L}$ and the peak power and energy of the laser pulse should also have reasonable values. Here, it is noted that $\tau_{L}$ is in the order of tens of ps and the energy of the laser pulse $U$ is $>1$ Joule for a QFEL operated in the $x$-ray regime. A laser undulator period of $1.064 \mu \mathrm{m}$ is a good choice as long pulse durations (ns-ps range) and relatively high-energy laser pulses are commercially available (e.g., Flash lamp-Pumped Nd:YAG laser with energy up to 25 Joules).

The experimental parameters for a QFEL experiment can now be obtained. The bunch length $L_{\mathrm{b}}=c Q / I$ is calculated and the condition $L_{\mathrm{b}} \gg L_{\mathrm{c}}$ for $\varepsilon_{n}$ is checked. In this work, the charge per bunch $Q$ is assumed using an approximate relation estimated for the TESLA FEL $r f$ 
photo-injector [16]:

$$
\varepsilon_{n} \approx 1.45\left(0.38 Q^{4 / 3}+0.095 Q^{8 / 3}\right)^{1 / 2},
$$

where the charge $Q$ is in $\mathrm{nC}$ and the normalized emittance $\varepsilon_{n}$ is in mm-mrad. Using Eq. (4) and the relation $\varepsilon_{n}=\sigma \sqrt{\Gamma\left(1+a_{0}^{2}\right)}$, the corresponding value of $I$ for $\varepsilon_{n}$ is calculated. $P$ and $a_{0}$ are chosen so that $L_{\mathrm{b}} \geq 50 L_{\mathrm{c}}$. The emittance may differ appreciably from that predicted by Eq. (11) if using other scaling laws such as $\varepsilon_{n} \approx 2.015 Q+0.024$ of [17]. From Eqs. (4) and (8), It is noted that the electron current varies with the square of the emittance. In this case even a small increase in the emittance could result in a significant increase in the electron current.

\subsection{Possible experimental parameters}

In the following examples, sub-Angstrom QFEL experiments at $\lambda_{r}=0.5 \AA, \lambda_{r}=0.3 \AA$, and $\lambda_{r}=0.2 \AA$ are demonstrated in the quantum limit where $\bar{\rho}=0.2$. In this sub-Angstrom region, major practical requirements are to use ultra-cold electron beam and a very low bunch charge $(<10 \mathrm{pC})$ that is required to obtain an ultra-low emittance ( $<0.1 \mathrm{~mm}$.mrad). The four independent parameters $\lambda_{L}, P, a_{0}$, and $n_{i}$, have been chosen satisfying the optimization criteria described in the above section.

Using Eq. (8) for $0<\sigma \leq R_{0}$, the dependences of the current $I$ on both the electron beam radius $\sigma$, and its normalized emittance $\varepsilon_{n}$ are shown in figures $1(\mathrm{a})$ and $1(\mathrm{~b})$ respectively. Using Eq. (9), the variation of $I$ against the peak power of FEL radiation $P_{r}$, is shown in figure 2.

To select an experimental operating point for $\bar{\rho}=0.2, Q$ is calculated for an applicable value of $\varepsilon_{n}$ using Eq. (11) (i.e., $\mathrm{Q}=1.2 \mathrm{pC}$ when $\varepsilon_{n}=0.01 \mathrm{~mm}$.mrad). Figures $1(\mathrm{a})$ and $1(\mathrm{~b})$ are used to select a suitable value of emittance seen to be $\varepsilon_{n}<0.05 \mathrm{~mm}$.mrad. In practice, in order to achieve such low emittance and low rms radius of the electron beam, the $\beta$-function must be small (few tens of $\mathrm{mm}$ ) at the interaction region. This requires strong electron focusing optics. In our work, the bunch length $L_{\mathrm{b}}$ is determined by $I$ and $Q$ (corresponding to $\varepsilon_{n}$ ) and should satisfy the condition $L_{\mathrm{b}} \gg L_{\mathrm{c}}$. Other parameters can be calculated from the equations listed in section 2 (or from figures 1 and 2). In Table 1, three parameter sets for the electron beam and laser undulator are given for $\lambda_{r}=0.5 \AA, 0.3 \AA$, and $0.2 \AA$. In Table 1 , it is also shown that the photon number per pulse is $\sim 10^{7}$ photons/pulse. For a modest $10 \mathrm{~Hz}$ repetition rate pulsed laser, the photon flux is predicted to reach a maximum of $\approx 10^{8}$ photons $/ \mathrm{sec}$. This value of photon flux is sufficient for many $x$-ray applications. It is noted that to make a sub-Angstrom FEL operating with a laser undulator in the classical regime where $\bar{\rho} \gg 1$ (e.g., $\bar{\rho}=5$ ), the current required becomes impractically high (e.g., I $>50 \mathrm{kA}$ ). So that operation of laser undulator $\mathrm{x}$-ray FELs in the quantum regime may be a more attractive option. 


\subsection{Numerical simulations}

QFEL simulations were performed using the model described in [5], where the wavefunction, $\Psi\left(\bar{z}, \bar{z}_{1}, \theta\right)$, which describes the electron pulse is expanded in terms of momentum states

$$
\Psi\left(\bar{z}, \bar{z}_{1}, \theta\right)=\frac{1}{\sqrt{2 \pi}} \sum_{n=-\infty}^{\infty} c_{n}\left(\bar{z}, \bar{z}_{1}\right) e^{i n \theta} .
$$

where $\theta=\left(k+k_{L}\right) z-c k t, \bar{z}=z / L_{g}, \bar{z}_{1}=(z-v t) / \beta L_{c}$, and $\left|c_{n}\left(\bar{z}, \bar{z}_{1}\right)\right|^{2}$ is the probability of finding an electron in a momentum state, $n$, at $\bar{z}$ and $\bar{z}_{1}$, where $\bar{z}$ and $\bar{z}_{1}$ are the scaled interaction distance and the electron bunch coordinate, respectively. Here, $\beta=v / c$ is the normalized average electron speed in the $z$-direction. In [5], it has also shown that the quantum-mechanical dynamics of FELs can be described by a Schrödinger equation coupled with the equation for a scaled field amplitude:

$$
\begin{array}{r}
i \frac{\partial \Psi(\bar{z}, \theta)}{\partial \bar{z}}=-\frac{1}{2 \bar{\rho}} \frac{\partial^{2} \Psi(\bar{z}, \theta)}{\partial \theta^{2}}-i \bar{\rho}\left(A e^{i \theta}-A^{*} e^{-i \theta}\right) \Psi(\bar{z}, \theta), \\
\frac{\partial A\left(\bar{z}, \bar{z}_{1}\right)}{\partial \bar{z}}+\frac{\partial A\left(\bar{z}, \bar{z}_{1}\right)}{\partial \bar{z}_{1}}=\int_{0}^{2 \pi}\left|\Psi\left(\bar{z}, \bar{z}_{1}, \theta\right)\right|^{2} e^{-i \theta} d \theta+i \delta A\left(\bar{z}, \bar{z}_{1}\right) .
\end{array}
$$

Substituting $\Psi\left(\bar{z}, \bar{z}_{1}, \theta\right)$ as defined in Eq. (12) in Eqs. (13) and (14), we obtain:

$$
\begin{aligned}
& \frac{\partial c_{n}}{\partial \bar{z}}=-i \frac{n^{2}}{2 \bar{\rho}} c_{n}-\bar{\rho}\left[A c_{n-1}-A^{*} c_{n+1}\right] . \\
& \frac{\partial A}{\partial \bar{z}}+\frac{\partial A}{\partial \bar{z}_{1}}=\sum_{n=-\infty}^{\infty} c_{n} c_{n-1}^{*}+i \delta A .
\end{aligned}
$$

where $\delta=\left(\gamma_{0}-\gamma\right) / \gamma \rho$ is the detuning parameter, $\rho|A|^{2}=\left(\varepsilon_{0}|E|^{2}\right) /\left(n \gamma m c^{2}\right)$ is the ratio between the energy density of radiation and of the resonant electron beam and the bunching parameter $b$ is defined as

$$
b=\sum_{n=-\infty}^{\infty} c_{n} c_{n-1}^{*} .
$$

The scaled average radiation energy $E$ emitted by the electron pulse is calculated using

$$
E(\bar{z})=\frac{1}{L_{b}} \int_{0}^{L_{b}+\bar{z}}\left|A\left(\bar{z}, \bar{z}_{1}\right)\right|^{2} d \bar{z}_{1} .
$$

Due to the propagation effect (where $c>v$ ), the radiation slips forward with respect to the electron bunch. In our case of $L_{\mathrm{b}} \gg L_{\mathrm{c}}$ (i.e., long electron bunch), the propagation effect is significant. In the bunch coordinate $\bar{z}_{1}$, the slippage distance of the radiation equals to $\bar{z}$. Therefore, the upper limit in the integration shown in Eq. (18) is $L_{b}+\bar{z}$.

In what follows, we investigate the evolution of the scaled field intensity $|A|^{2}$ as a function of $\bar{z}_{1}$ by solving Eqs. (15) and (16) numerically. The corresponding power spectra are also 
depicted to show the degree of radiation coherence. Our simulations assume that almost all electrons are initially in the momentum state $n=0$ and the effects of intrinsic energy spread are neglected. In order to neglect the intrinsic energy spread, it should be smaller than the FEL line width $\Gamma=\rho \sqrt{\bar{\rho}}$. For the parameters shown in Table I, our assumption is met when the energy spread is approximately $<3 \mathrm{keV}$. A further increase in the energy spread will contribute to the broadening of the resonance, and results in gain degradation. The initial conditions for all simulations are $A\left(\bar{z}=0, \bar{z}_{1}\right)=0, \quad c_{-1}\left(\bar{z}=0, \bar{z}_{1}\right)=b_{0} e^{i \phi\left(\bar{z}_{1}\right)}$ where $\phi\left(\bar{z}_{1}\right)$ is a randomly fluctuating phase with values in the range $[0,2 \pi]$, and $c_{0}\left(\bar{z}=0, \bar{z}_{1}\right)=\sqrt{1-b_{0}^{2}}$. Using Eq. (17), $\left|b\left(\bar{z}=0, \bar{z}_{1}\right)\right| \approx b_{0}$. In order to estimate an initial value of the bunching parameter $b_{0}$, we recall the classical expression of the bunching parameter $b$ that is [18]

$$
b=\frac{1}{N} \sum_{j=1}^{N} e^{-i \theta} .
$$

where $N$ is the number of electrons in one radiation wavelength. For an initially random distribution of electrons $\left|b_{0}\right|^{2}=1 / N=\left(L_{b} / \lambda_{r}\right) /(Q / e)$. Using the parameters in our examples, $b_{0} \sim 0.08$. This discussion assumes a classical picture of the electron beam and of the electromagnetic radiation. The quantum fluctuations in the position and momentum of the electron beam would be expected to reduce of in the classical shot noise level. As a first estimate, we assume $b_{0}=0.01$ in our numerical examples.

For the parameters of set (1) when $\lambda_{r}=0.5 \AA$ shown in table 1 , the top plots in figures $3(a), 3(b)$, and 3 (c) show the scaled field intensity $|A|^{2}$ vs. $\bar{z}_{1}$ at different values of $\bar{z}$. The bottom plots show the corresponding scaled power $P$ as a function of a scaled frequency $\bar{\omega}=(\omega-\omega) / 2 \rho \omega$ where $\omega$ is the resonance frequency. Figure $3(\mathrm{~d})$ shows the variation of the average energy $E$ given by Eq. (18) as a function of $\bar{z}$. Similar simulations are carried out for the parameters of set (3) when $\lambda_{r}=0.2 \AA$ and are shown in figures 4(a), 4(b), 4(c), and 4(d), respectively. One significant difference between Set (1) and Set (3) is the difference in the value of $L_{b} / L_{c}=81.2$ and 140.4 in Set (1) and Set (3) respectively. It will be shown that the bunch length has a small influence on our numerical simulations.

During the lethargy regime seen in figure 3(d) and figure 4(d) (e.g., when $\bar{z}=1.4$ ), figure $3(a)$ and figure 4(a) show that the fields have many random fluctuations along the bunch length. Correspondingly, the spectra have random spikes at different wavelengths. In the top plots of figure 3(a) and figure 4(a), the edge of the scaled bunch length $\bar{z}_{1}=L_{b} / L_{c}$ is illustrated by the vertical dashed line. At the beginning of the exponential growth regime (e.g., when $\bar{Z}=10.1$ ), figure $3(\mathrm{~b})$ and figure $4(\mathrm{~b})$ show that the power spectra have narrower width and the spectral characteristics are significantly improved. In the regime at the end of the interaction length $(\bar{z}=24.4)$, the radiation has an extremely narrow bandwidth as shown in figure $3(\mathrm{c})$ and figure $4(\mathrm{c})$. Note that the ratio $L_{b} / L_{c}$ for Set (3) is greater than that for of Set (1). Consequently, the spectrum of Set 3 shown in fig. 4(c) has larger number of random spikes than fig. 3(c) due to 
the statistical nature of the SASE process [8]. Figure 3(b) and figure 4(b) interestingly show that narrow quantum spectra can be realized, not only at the saturation regime $(\bar{z}=24.4)$, but also at the exponential regime (e.g., $\bar{z} \sim 10$ ). It can be seen that the radiation line widths at halfmaximum are $\sim 2 \rho$ and $\sim \rho$ when $\bar{z} \sim 10$ and $\bar{z} \sim 24.4$, respectively. This result permits relaxation of the experimental constraints on sub-Angstrom FEL using a relatively low-quality electron beam.

\section{Conclusions}

Using a Compton backscattering scheme, we have described the design of proposed a sub-Angstrom FEL operating in the quantum SASE mode. The practical constraints and optimum conditions for efficient operation of QFELs are discussed. It was shown that the main requirement is very high beam quality. Therefore, it is desirable to operate at low charge (in $\mathrm{pCs}$ range) to generate low emittance, low energy spread, and short bunch lengths. While beam degradation effects due to space charge as the QFEL interaction proceeds have not been modelled here, they have been considered in [19-21] and do not appear to be a factor that cannot be managed. We numerically demonstrate that the QFEL has a number of potentially attractive features, including extremely narrow bandwidth, short pulse structure, and high photon flux. This study suggests that a compact QFEL source in the sub-Angstrom range should be experimentally realizable using current laser and electron beam technologies.

\section{Acknowledgements}

The authors would like to acknowledge support from: The British Council and the Science and Technology Development Fund of the Egyptian State Ministry for Scientific

Research for their support; Science and Technology Facilities Council Agreement Number 4163192 Release \#3; ARCHIE-WeSt HPC, EPSRC grant EP/K000586/1; and EPSRC Grant $\mathrm{EP} / \mathrm{M} 011607 / 1$.

\section{References}

[1] E. S. Sarachik and G. T. Schappert, Classical theory of the scattering of intense laser radiation by free electrons, Phys. Rev. D 1 (1970) 2738-2753.

[2] P. Sprangle, A. Ting, E. Esarey, and A. Fisher, Tunable, short pulse hard x-rays from a compact laser synchrotron source, J. Appl. Phys. 72 (1992) 5032-5038.

[3] K. J. Kim, Characteristics of Synchrotron Radiation, AIP Conf. Proc. 184 (AIP, New York), vol. I (1989) 565-632. 
[4] N. Piovella, M. Gatelli, and R. Bonifacio, Quantum effects in the collective light scattering by coherent atomic recoil in a Bose-Einstein condensate, Opt. Commun. 194 (2001) 167-173.

[5] R. Bonifacio, N. Piovella, G. R. M. Robb, and M. M. Cola, Propagation effects in the quantum description of collective recoil lasing, Opt. Commun. 252 (2005) 381-396.

[6] R. Bonifacio, N. Piovella, G. R. M. Robb, and A. Schiavi, Quantum regime of free electron lasers starting from noise, Phys. Rev. ST Accel. Beams 9 (2006) 090701-090709.

[7] R. Bonifacio, N. Piovella, M. M. Cola, and L. Volpe, Quantum regime of free electron lasers starting from noise, Nucl. Instr. and Meth. A 577 (2007) 745-750.

[8] R. Bonifacio, I. De Salvo, P. Pierini, N. Piovella, and C. Pelleggrini, Spectrum, temporal structure, and fluctuations in a high-gain free-electron laser starting from noise, Phys. Rev. Lett. 73 (1994) 70-73.

[9] W. P. Leemans et al., GeV electron beams from a centimetre-scale accelerator, Nature Phys. 2 (2006) 696-699.

[10] W. P. Leemans et al., Multi-GeV Electron Beams from Capillary-Discharge-Guided Subpetawatt Laser Pulses in the Self-Trapping Regime, Phys. Rev. Lett 113 (2014) 245002-245006.

[11] I. V. Pogorelsky, I Ben-zvi, and T. Hirose, Laser-electron Compton Interaction in plasma channels, BNL Report No. 65907 (1998).

[12] Li. Dongguo, K. Yokoya, T. Hirose, and R. Hamatsu, Laser Propagation and Compton scattering in parabolic channel, Jpn. J. Appl. Phys. 42 (2003) 1800-1806.

[13] S. Jackel et al., Channeling of terawatt laser pulses by use of hollow waveguides, Opt. Lett. 20, (1995) 1086-1088.

[14] M. Borghesi et al., Guiding of a 10-TW picosecond laser pulse through hollow capillary tubes, Phys. Rev. E 57, (1997) R4899-R4902.

[15] E. L. Saldin, E. Schneidmiller, and M. V. Yurkov, Self-amplified spontaneous emission FEL with energy-chirped electron beam and its application for generation of attosecond x-ray pulses, Phys. Rev. ST Accel. Beams 9 (2006) 050702-050707.

[16] J. Rosenzweig and E. Colby, Charge and Wavelength Scaling of RF Photoinjectors: A Design Tool, in Proceedings of the 1995 Particle Accelerator Conference, IEEE Publishing, Piscataway, New Jersey (1996) 957-960.

[17] F. Zhou, Emittance studies for the TTF FEL Photoinjector, Desy FlashTesla FEL- Report 02 (1999). 
[18] B. W. J. McNeil, M. W. Poole, and G. R. M. Robb, Unified model of electron beam shot noise and coherent spontaneous emission in the helical wiggler free electron laser, Phys. Rev. ST Accel. Beams 6 (2003) 070701-070711.

[19] S. B. van der Geer, E. J. D. Vredenbregt, O. J. Luiten and M. J. de Loos, An ultracold electron source as an injector for a compact SASE-FEL, J. Phys. B: At. Mol. Opt. Phys. 47 (2014) 234009-234016.

[20] B. Zeitler, K. Floettmann, and F. Grüner, Linearization of the longitudinal phase space without higher harmonic field, Phys. Rev. ST Accel. Beams 18 (2015) 120102-120117.

[21] J.B. Rosenzweig, et al., Next generation high brightness electron beams from ultra-high field cryogenic radiofrequency photocathode sources.

http://arxiv.org/pdf/1603.01657v2.pdf 


\section{Figures and Tables}

Figure 1

(a)

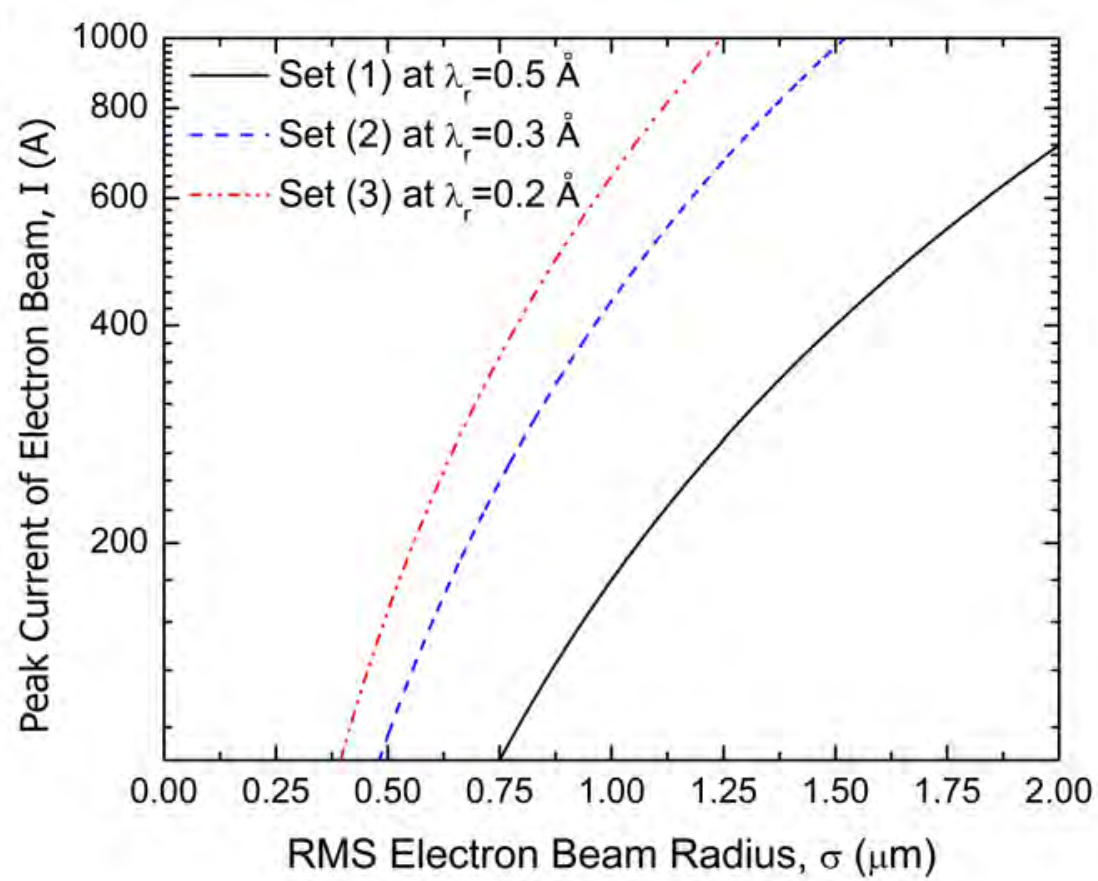

(b)

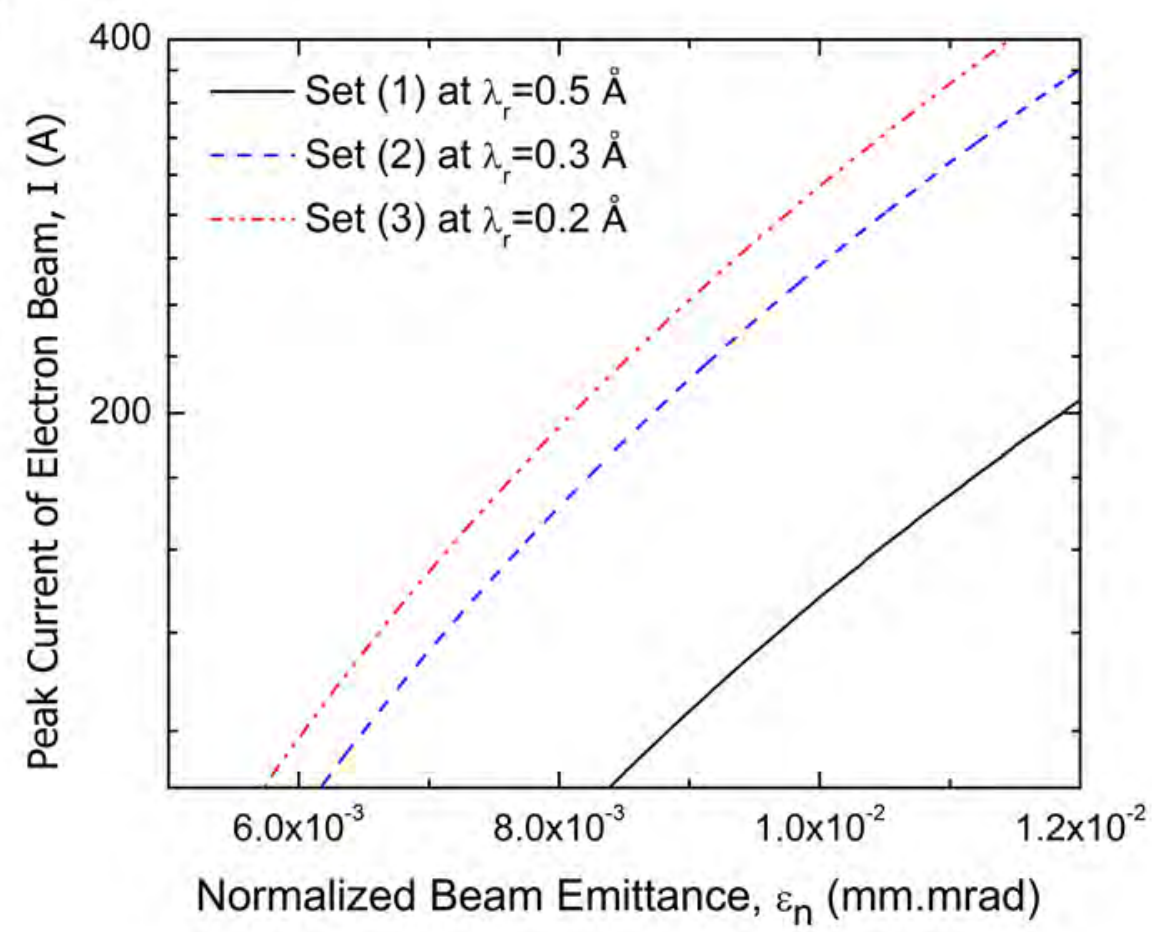

Figure 1. (a) The electron beam current $I$ vs. $\sigma$ using Eq. (4). (b) The electron beam current $I$ vs. $\varepsilon_{n}=\sigma \sqrt{\Gamma\left(1+a_{0}^{2}\right)}$. 


\section{Figure 2}

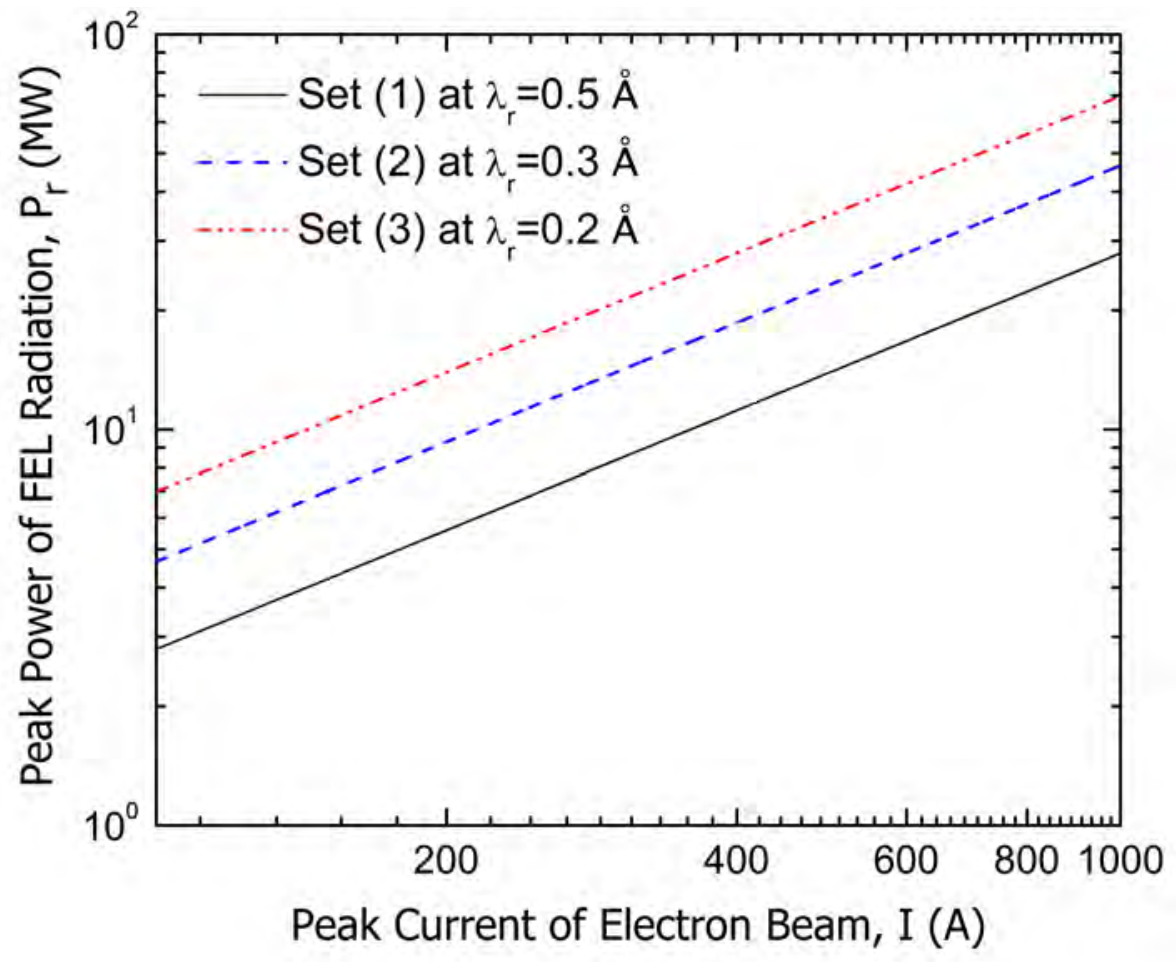

Figure 2. The peak current of electron beam $I$ is plotted against the peak power of FEL radiation $P_{r}$ using Eq. (10). 
Table 1. Possible experimental operating parameters. Three parameters sets are shown at $\lambda_{r}=0.5 \AA, \lambda_{r}=0.3 \AA$, and $\lambda_{r}=0.2 \AA$.

\begin{tabular}{|lllll|}
\hline & & Set (1) & Set (2) & Set (3) \\
\hline Radiation wavelength & $\lambda_{r}(\AA)$ & 0.5 & 0.3 & 0.2 \\
\hline QFEL parameter & $\bar{\rho}$ & 0.2 & 0.2 & 0.2 \\
\hline Laser wavelength & $\lambda_{L}(\mu \mathrm{m})$ & 1.064 & 1.064 & 1.064 \\
\hline Laser power & $P(\mathrm{TW})$ & 0.6 & 0.9 & 1.75 \\
\hline Laser minimum radius & $R(\mu \mathrm{m})$ & 13.071 & 11.60 & 10.74 \\
\hline Laser pulse width & $\tau_{L}(\mathrm{ps})$ & 13.31 & 10.61 & 9.1 \\
\hline Laser pulse energy & $E(\mathrm{Joule})$ & 8.07 & 9.55 & 15.92 \\
\hline$\left(c \tau_{L} / L_{g}\right)$ & $n_{i}$ & 24.4 & 24.4 & 24.4 \\
\hline Laser parameter & $a_{0}$ & 0.214 & 0.2952 & 0.4445 \\
\hline Classical FEL parameter & $\rho$ & $1.28 \times 10^{-4}$ & $1.62 \times 10^{-4}$ & $1.90 \times 10^{-4}$ \\
\hline Classical gain length & $L_{g}(\mathrm{~mm})$ & 0.33 & 0.26 & 0.223 \\
\hline Classical cooperation length & $L_{c}(\mathrm{~nm})$ & 31.03 & 14.70 & 8.40 \\
\hline E-beam energy & $\gamma$ & 74.58 & 98.18 & 126.20 \\
\hline Charge per electron bunch & $Q(\mathrm{pc})$ & 1.2 & 1.2 & 1.2 \\
\hline Normalized emittance & $\varepsilon_{n}(\mathrm{~mm} . \mathrm{mrad})$ & 0.01 & 0.01 & 0.01 \\
\hline E-beam rms radius & $\sigma(\mu \mathrm{m})$ & 0.89 & 0.78 & 0.68 \\
\hline E-beam bunch length & $L_{b}(\mu \mathrm{m})$ & 2.52 & 1.37 & 1.18 \\
\hline E-beam peak current & $I(\mathrm{kA})$ & 0.142 & 0.263 & 0.304 \\
\hline Radiation Peak power & $P_{r}(\mathrm{MW})$ & 3.98 & 12.24 & 21.26 \\
\hline Gain bandwidth & $\Gamma$ & $5.75 \times 10^{-5}$ & $7.28 \times 10^{-5}$ & $8.50 \times 10^{-5}$ \\
\hline FEL linewidth & $\Delta \omega / \omega$ & $1.98 \times 10^{-5}$ & $2.18 \times 10^{-5}$ & $1.69 \times 10^{-5}$ \\
\hline Number of spikes & $N_{s}$ & 1 & 1 & 1 \\
\hline Photon numbers per pulse & $N_{p h}$ & $9.0 \times 10^{6}$ & $9.0 \times 10^{6}$ & $9.0 \times 10^{6}$ \\
\hline Normalized bunch length & $L_{b} / L_{c}$ & 81.20 & 93.15 & 140.44 \\
\hline
\end{tabular}


Figures 3
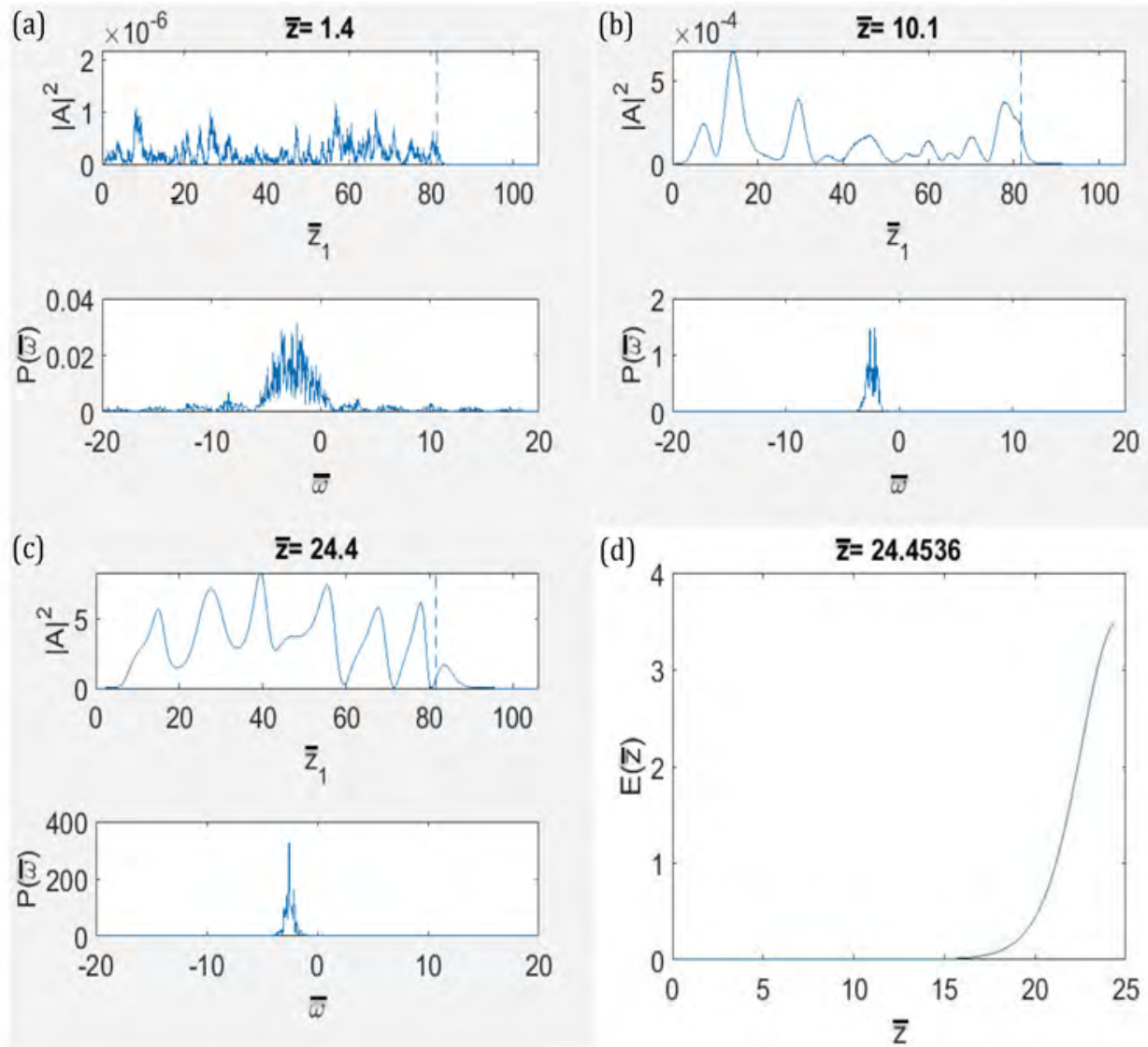

Figure 3. For Set (1) at $\lambda_{r}=0.5 \AA,|A|^{2}$ as a function of $\bar{z}_{1}$ (top plots) and the corresponding scaled power $P$ as a function of scaled frequency $\bar{\omega}=(\dot{\omega}-\omega) / 2 \rho \omega$ (bottom plots) at (a) $\bar{z}=1.4$ (at the lethargy region), (b) $\bar{z}=10.1$ (at the exponential region), and (c) full interaction length $\bar{z}=24.4$ (at the saturation region) when $L_{b} / L_{c}=81.2$. Fig. 3 (d) shows the variation of the scaled radiation energy against the interaction length $\bar{z}$. 
Figures 4
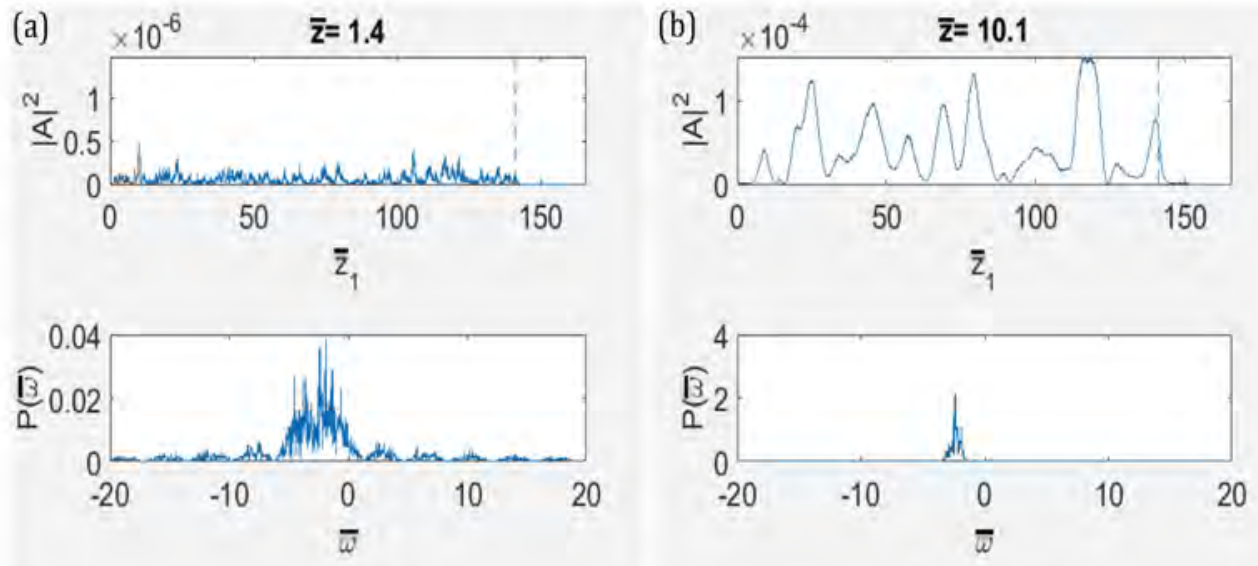

(c)
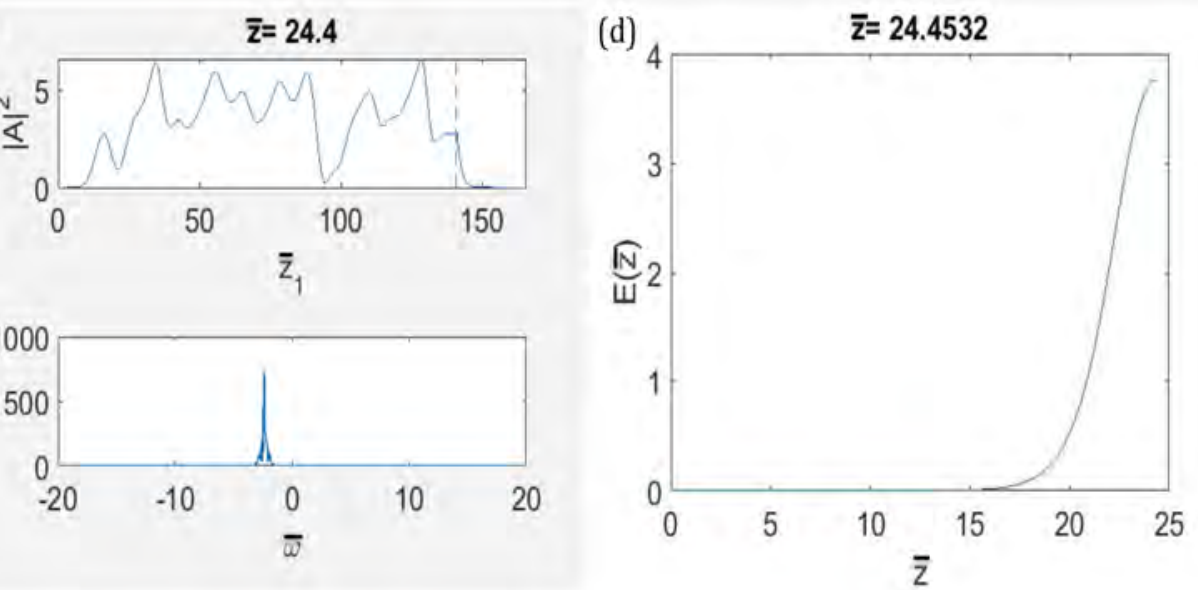

Figure 4. For Set (3) at $\lambda_{r}=0.2 \AA,|A|^{2}$ vs. $\bar{z}_{1}$ (top plots) and $P$ vs. $\bar{\omega}$ (bottom plots) at (a) $\bar{z}=1.4$ (at the lethargy region), (b) $\bar{z}=10.1$ (at the exponential region), and (c) full interaction length $\bar{z}=24.4$ (at the saturation region) when $L_{b} / L_{c}=140.44$. Fig. 4 (d) shows the variation of $E$ against $\bar{z}$. 